\title{
UNA ESPECIE NUEVA DE ACIDOCROTON SECCIÓN OPHELLANTHA (EUPHORBIACEAE) DE TABASCO, MÉXICO
}

\author{
Ana M. Hanan-Alipl ${ }^{1,3}$ y Victor W. Steinmann ${ }^{2}$ \\ ${ }^{1}$ Universidad Autónoma de Nayarit, Programa de Biología, Unidad Académica de \\ Agricultura, km 9 carretera Tepic-Compostela, 63155 Tepic, Nayarit, México. \\ ${ }^{2}$ Instituto de Ecología, A.C., Centro Regional del Bajío, Apdo. postal 386, 61600 \\ Pátzcuaro, Michoacán, México. \\ ${ }^{3}$ Autor para la correspondencia: hananalipi@yahoo.com.mx
}

\section{RESUMEN}

Se describe e ilustra a Acidocroton madrigalensis, una nueva especie del estado de Tabasco, México. Se conoce solamente de la Sierra El Madrigal y el Cerro La Campana, en donde es un componente abundante del estrato bajo en la selva alta perennifolia sobre afloramientos de roca caliza. Pertenece a la sección Ophellantha y se caracteriza por sus diminutas estípulas no espiniformes, hojas grandes y cortamente pecioladas y por tener flores estaminadas con 20 a 35 estambres. Se presenta un cuadro mostrando las diferencias principales entre A. madrigalensis y las otras dos especies del género conocidas de México, A. spinosus y A. steyermarkii.

Palabras clave: Acidocroton, Euphorbiaceae, México, sección Ophellantha, Tabasco.

\begin{abstract}
The new species Acidocroton madrigalensis is described and illustrated from the state of Tabasco, Mexico. It is known only from the Sierra El Madrigal and the Cerro La Campana, where it is a frequent component of the under story of tropical rain forest, occurring on limestone outcrops. It belongs to section Ophellantha and is characterized by its diminutive, non-spinous stipules, large leaves that are shortly petiolate, and staminate flowers with 20 to 35 stamens. A table is presented that provides the principal differences among $A$. madrigalensis and the other two species of the genus present in Mexico, $A$. spinosus y A. steyermarkii.
\end{abstract}


Key words: Acidocroton, Euphorbiaceae, Mexico, section Ophellantha, Tabasco.

El bosque tropical perennifolio o selva alta perennifolia es el tipo de vegetación más exuberante que existe en la Tierra (Rzedowski, 1978). En el estado de Tabasco, al igual que en muchos otros lugares, esta vegetación ha sido fuertemente afectada por actividades humanas. De hecho, dentro de la entidad existen pocos sitios donde todavía permanece en forma relativamente bien conservada; uno de ellos es la Sierra El Madrigal en el municipio de Teapa, ubicado en el sur del estado. Durante una serie de exploraciones botánicas que se llevaron a cabo en esta sierra (Hanan, 1997; Pérez et al., 2005) se encontró una especie de Acidocroton Griseb., que fue detectada como nueva por primera vez por el Dr. Grady Webster (com. pers. 1997) y el propósito de este artículo es proponerla formalmente como:

Acidocroton madrigalensis A. Hanan \& V.W. Steinm. sp. nov. Tipo: MÉXICO, Tabasco, municipio Teapa, Sierra El Madrigal, a $0.7 \mathrm{~km}$ al SE del edificio principal del Centro Regional Tropical Puyacatengo, 17³1'30.25" N, 9255'26.28" W, 325 m, 07 abr 1993, A. Hanan y C. Collado 692 (holotipo MEXU; isotipos IEB, UJAT). Fig. 1.

Arbor usque ad $5 \mathrm{~m}$ alta, monoecia; latex translucens rubescens; stipulae inconspicuae, $0.4-0.7 \mathrm{~mm}$ longae; folia alterna, petioli breves, laminae foliorum ellipticae vel oblongae, 6-17 cm longae, $2.5-9 \mathrm{~cm}$ latae, apice acuminato interdum falcato; inflorescentia staminata racemosa, 1-3 cm longa; flororum staminatorum pedicellis $0.5-1.5 \mathrm{~mm}$ longis, sepala 5 , ovata vel orbicularia, $0.5-0.7 \mathrm{~mm}$ longa, petala 5, ovata vel orbicularia, $5 \mathrm{~mm}$ longa, discus globosus pubescens, stamina 20-35, filamenta $1 \mathrm{~mm}$ longa; flores pistillati solitarii, pedicellis $0.5-1 \mathrm{~cm}$ longis, sepala (4)5, lanceolata, 5-12 mm longa, accrescentia, petala absentia, discus strigoso-hirsutus, styli 3, crassi, plani, 6-7 mm longi, adpressi; capsula sphaerica, $1 \mathrm{~cm}$ diametro, glabra.

Árbol monoico de hasta $5 \mathrm{~m}$ de alto; látex traslúcido tornándose rojo, ramas delgadas, teretes, estrigulosas; estípulas inconspicuas, endurecidas, cónicas, de 0.4 a $0.7 \mathrm{~mm}$ de largo, estrigulosas; hojas alternas; pecíolo estrigoso principalmente en la superficie abaxial, de 3 a 5(-10) mm de largo; lámina membranácea, verde en el haz, más pálida en el envés, elíptica a ligeramente obovada, de 6 a $17 \mathrm{~cm}$ de largo, de 2.5 a $9 \mathrm{~cm}$ de ancho, acuminada en el ápice a veces éste ligeramente curvado, margen estrigoso, ligeramente revoluto, entero o a veces remotamente glandular-denticulado, base atenuada, pubescencia muy escasa en el haz con algunos pelos adpresos gene- 




Fig. 1. Acidocroton madrigalensis Hanan \& V.W. Steinm. A. rama con hojas y flores; B. tallo estriguloso con estípula; C. margen de la hoja, glandular-denticulado; D. inflorescencia masculina con botones; E. flor estaminada; F. estambre; G. inflorescencia masculina y flor pistilada, mostrando sépalos, disco estrigoso-hirsuto, ovario y estilos bífidos; H, I, J. distintos ovarios vistos desde arriba, mostrando la variación en la división de los ápices de sus estilos; $\mathrm{K}$. fruto con cáliz acrescente y estilos persistentes; L. restos del fruto: columela y cáliz; M y N. cocos monospermos bivalvados luego de liberar la semilla, vista dorsal y ventral respectivamente. Las ilustraciones A, B, C, D, se basan en A. Hanan y C. Collado 389 (MEXU); E, F, G, J, en A. Hanan et al. 692 (MEXU, tipo); H, en A. Hanan et al. 911 (MEXU); I, K, L, M, N, en A. Hanan et al. 910 (MEXU). 
ralmente sobre las venas, el envés con la vena media estrigosa, venación ligeramente elevada en el envés, nervios laterales (4-)5-6(-7) pares; inflorescencias masculinas racimos de 1 a $3 \mathrm{~cm}$ de largo, opuestos o casi opuestos a las hojas, a veces terminales, con 1 a 2 flores en antesis y 3 a 5 botones; flores estaminadas con pedicelos de 0.5 a $1.5 \mathrm{~cm}$ de largo, estrigosos, brácteas triangulares, glandular-dentadas, estrigosas, de ca. $1 \mathrm{~mm}$ de largo, persistentes en el eje de la inflorescencia; bractéolas parecidas a las brácteas pero más pequeñas; cáliz campanulado, sépalos 5 , ovados a orbiculares, de 1.5 a 2 veces más anchos que largos, de 0.5 a $0.7 \mathrm{~mm}$ de largo, de 0.8 a $1.2 \mathrm{~mm}$ de ancho, ápice obtuso, pelos adpresos en la superficie abaxial; pétalos 5, libres, orbiculares a ligeramente ovados, blancos, de ca. $5 \mathrm{~mm}$ de largo, de 3.5 a $4.5 \mathrm{~mm}$ de ancho, glabriúsculos; glándulas pubescentes presentes entre los estambres; estambres 20 a 35, erectos, introrsos, filamentos de ca. $1 \mathrm{~mm}$ de largo, anteras basifijas, el apéndice del conectivo triangular; flores pistiladas solitarias, en pedicelos de 0.5 a $1.5 \mathrm{~cm}$ de largo, estrigosos, brácteas triangulares, glandular-dentadas, estrigosas, de 1.2 a $7.5 \mathrm{~mm}$ de largo; bractéolas parecidas a las brácteas, de 0.7 a $1.2 \mathrm{~mm}$ de largo; sépalos 5 o a veces 4 pero entonces uno bífido, a veces unidos en la base, acrescentes, foliáceos, lanceolados, de 5 a $12 \mathrm{~mm}$ de largo y de 2 a 4.5(-5.7) $\mathrm{mm}$ de ancho (los límites mayores casi en fruto), margen estrigoso y glandular-dentado; pétalos ausentes; disco rodeando al ovario, estrigoso-hirsuto; ovario tricarpelar, glabro; estilos 3, gruesos, aplanados, de 6 a $7 \mathrm{~mm}$ de largo, adpresos al ovario, sus ápices variando de bífidos a dos veces bífidos o laciniados, con pelos adpresos en la superficie abaxial; placentación axilar, un óvulo por lóculo; fruto una cápsula esférica, tricarpelar, verde, glabra, de aproximadamente $1 \mathrm{~cm}$ de diámetro, con el cáliz acrescente y los estilos persistentes, separándose en 3 cocos monospermos bivalvados, columela persistente; semillas desconocidas.

Material adicional examinado. MÉXICO. Tabasco: municipio de Teapa, Sierra El Madrigal, a $1.4 \mathrm{~km}$ al SE del edificio principal del Centro Regional Tropical Puyacatengo, 17³1'19.02" N, 9255'05.37" W, 420 m, 24 abr 1991, A. Hanan y C. Collado 389 (FCME, IEB, MEXU, UJAT); Sierra El Madrigal, 16 oct 1991, $A$. Hanan y C. Collado 568 (MEXU); Sierra El Madrigal, en "El Tanque" a $0.57 \mathrm{~km}$ al NE del edificio principal del Centro Regional Tropical Puyacatengo, 17 $31^{\circ} 51.53$ " N, 9255'34.62" W, 07 oct 1992, A. Hanan et al. 676 (UJAT); Sierra El Madrigal a $0.66 \mathrm{~km}$ al E del edificio principal del Centro Regional Tropical Puyacatengo, 08 abr. 1993, A. Hanan 706 (IEB); Sierra El Madrigal, 13 mar 1997, A. Hanan et al. 910 (MEXU, UJAT), 911 (FCME, IEB, MEXU, UJAT); Cerro del Madrigal, 215 m, 29 oct 1986, A. Guadarrama et al. 949 (IEB); Cerro las Campanas $3 \mathrm{~km}$ E of Teapa, ca. $50 \mathrm{~km} \mathrm{~S}$ of Villahermosa, 12 ago 1974, J. Conrad et al. 2876 (MEXU). 
Acidocroton madrigalensis se conoce de la Sierra El Madrigal y el Cerro La Campana, prolongaciones de la Sierra Norte de Chiapas ubicadas al sur del estado de Tabasco, en donde es un componente abundante del estrato bajo en la selva alta perennifolia que ahí se desarrolla, sobre grandes afloramientos de roca caliza con fuerte desarrollo cárstico. Crece en elevaciones de $215 \mathrm{~m}$ a $420 \mathrm{~m}$. Se le ha encontrado en floración de febrero a abril y de agosto a octubre. Debido a su distribución limitada y a las amenazas a las que está sujeta la selva alta perennifolia se considera vulnerable a la extinción.

Acidocroton es un género neotropical que incluye aproximadamente 15 especies con su mayor diversidad en las Antillas (Govaerts et al., 2000). Además de la nueva especie, otras dos se encuentran en México: A. spinosus (Standl.) G.L. Webster y A. steyermarkii (Standl.) G.L. Webster. Ambas fueron descritas originalmente en el género Ophellantha Standl., pero Webster (1994) lo redujo a una sección de Acidocroton (sec. Ophellantha (Standl.) G.L. Webster); sin embargo, posteriormente Radcliffe-Smith (2001) los reconoció como géneros distintos. Se decidió seguir la propuesta de Webster y tratar este grupo como sección de Acidocroton, porque los rasgos vegetativos no son tan marcados como para justificar el reconocimiento de dos géneros distintos. Además, las diferencias entre Ophellantha y Acidocroton enlistadas por Radcliffe-Smith (op. cit.) no siempre son absolutas. Por ejemplo, $A$. spinosus y A. steyermarkii, ambas ubicadas en Ophellantha según este autor, tienen menos de 50 estambres, poseen estípulas a menudo marcadamente espinosas, y los ovarios pueden tener 3 carpelos, aunque en el caso de $A$. spinosus por lo general son dos. Sin embargo, todas estas características según Radcliffe-Smith son de Acidocroton y no de Ophellantha. Por otro lado, los análisis filogenéticos de la familia basados en caracteres moleculares (Berry et al., 2005; Wurdack et al., 2005) incluyen algunas especies de Acidocroton y Ophellantha, pero la muestra no se consideró suficiente para concluir sobre la monofilia de estos taxa. Se agrupan en un clado estrechamente vinculado con el género Croton L., de la tribu Crotoneae y la relación entre estos grupos requiere un estudio más amplio.

La nueva especie se puede distinguir de las otras dos de Acidocroton presentes en México por características foliares como: peciolos relativamente cortos ((3-)4(-10) vs. 10-14 mm), láminas más grandes (6-17 x 2.5-9 vs. 5-9 x 2.5-5.5 cm) y estípulas relativamente pequeñas (0.4-0.7 vs. $2-5 \mathrm{~mm})$, no espinosas (vs. a menudo espinosas). Otras diferencias se encuentran en el Cuadro 1.

El nombre de la especie está dedicado a la Sierra El Madrigal, municipio de Teapa, Tabasco (17² $\left.25^{\prime} 15^{\prime \prime}-17^{\circ} 32^{\prime} 30^{\prime \prime} \mathrm{N}, 9^{\circ} 49^{\prime}-92^{\circ} 56^{\prime} \mathrm{W}\right)$, uno de los últimos reductos de la selva alta perennifolia en el estado, y que junto con la Sierra Norte de 
Cuadro 1. Principales diferencias entre las tres especies de Acidocroton presentes en México.

\begin{tabular}{|c|c|c|c|}
\hline & A. madrigalensis & A. spinosus & A. steyermarkii \\
\hline Estípulas espiniformes & no & a menudo & a menudo \\
\hline Estípulas, longitud (mm) & $0.4-0.7$ & $3-5$ & $2-2.5$ \\
\hline Pecíolos, longitud (mm) & $(3-) 4(-10)$ & $10-14$ & $10-13$ \\
\hline $\begin{array}{l}\text { Hojas, tamaño } \\
\text { largo } x \text { ancho }(\mathrm{cm})\end{array}$ & $6-17 \times 2.5-9$ & $5-9 \times 2.5-5.5$ & $5-6.5 \times 1.5-2.5$ \\
\hline $\begin{array}{l}\text { Láminas de las hojas, } \\
\text { forma }\end{array}$ & $\begin{array}{l}\text { elípticas a } \\
\text { ligeramente } \\
\text { obovadas }\end{array}$ & $\begin{array}{l}\text { elípticas a ovado- } \\
\text { elípticas }\end{array}$ & $\begin{array}{c}\text { lanceoladas a } \\
\text { lanceolado-oblongas }\end{array}$ \\
\hline $\begin{array}{l}\text { Flores estaminadas: } \\
\text { color de pétalos }\end{array}$ & blanco & verde & verde \\
\hline estambres, número & $20-35$ & 16-17 (hasta 50) & $\begin{array}{l}16 \text { (se conoce de una } \\
\text { sola flor en botón) }\end{array}$ \\
\hline filamentos, longitud (mm) & 1 & $3-5$ & $\begin{array}{l}\text { se desconoce en } \\
\text { antesis }\end{array}$ \\
\hline $\begin{array}{l}\text { Flores pistiladas: } \\
\text { margen de los sépalos }\end{array}$ & denticulado & denticulado & entero \\
\hline $\begin{array}{l}\text { lóculos del ovario y } \\
\text { estilos, número }\end{array}$ & 3 & $2(-3)$ & 3 \\
\hline Fruto, pubescencia & glabro & $\begin{array}{c}\text { ligeramente } \\
\text { adpreso, setuloso } \\
\text { o glabro }\end{array}$ & $\begin{array}{c}\text { densamente } \\
\text { velutino-pilósuloso }\end{array}$ \\
\hline
\end{tabular}

Chiapas, así como las regiones de Uxpanapa y Chimalapas, es conocida como "área del arco" o "crescent area", región que ha sido postulada por Wendt (1987a, 1993) como refugio florístico del Cenozoico con base en la evidencia paleobotánica y fitogeográfica, así como por el alto número de taxa endémicos (Hernández y HananAlipi, 1998; Monro, 1999; Schutzman et al., 1988; Wendt, 1987b; Zamudio, 1988) y poblaciones disyuntas.

\section{AGRADECIMIENTOS}

Agradecemos a Isabel Noriega Escurdia por la elaboración de la ilustración, a Héctor Cervantes Maya por hacernos llegar los ejemplares que se examinaron, a los herbarios FCME, IEB, MEXU y UJAT por el acceso a sus colecciones y a Grady L. 
Webster por proporcionar información importante sobre la nueva especie. El trabajo de Steinmann fue realizado con apoyo económico del Instituto de Ecología, A.C. (cuenta 20006), del Consejo Nacional de Ciencia y Tecnología y de la Comisión Nacional para el Conocimiento y Uso de la Biodiversidad.

\section{LITERATURA CITADA}

Berry, P. E., A. L. Hipp, K. J. Wurdack, B. Van Ee y R. Riina. 2005. Molecular phylogenetics of the giant genus Croton and the tribe Crotoneae (Euphorbiaceae sensu stricto) using ITS and trnL-trnF DNA sequence data. Amer. J. Bot. 92: 1520-1534.

Govaerts, R., D. G. Frodin y A. Radcliffe-Smith. 2000. World checklist and bibliography of Euphorbiaceae (and Pandaceae). Vols. 1-4. The Board of Trustees of the Royal Botanic Gardens. Kew, UK. 1622 pp.

Hanan, A. M. 1997. Análisis florístico de la Sierra El Madrigal, Teapa, Tabasco. Tesis Profesional. Facultad de Ciencias, Universidad Nacional Autónoma de México. México, D.F., México. 50 pp.

Hernández, H. M.y A. M. Hanan-Alipi. 1998. Zapoteca quichoi (Leguminosae, Mimosoideae), a new species from southeastern Mexico. Brittonia 50(2): 211-213.

Monro, A. K., 1999. Seven new species of Pilea Lindley (Urticaceae) from Mesoamerica. Novon 9: 390-400.

Pérez, L. A., M. Sousa S., A. M. Hanan, F. Chiang y P. Tenorio. 2005. Vegetación terrestre. Capítulo 4. In: Bueno, J., F. Álvarez y S. Santiago (eds.). Biodiversidad del estado de Tabasco. Instituto de Biología, Universidad Nacional Autónoma de México - Consejo Nacional para el Conocimiento y Uso de la Biodiversidad. México, D.F., México. pp. 65-110.

Radcliffe-Smith, A. 2001. Genera Euphorbiacearum. Royal Botanic Gardens. Kew, UK. $455 \mathrm{pp}$.

Rzedowski, J. 1978. Vegetación de México. Editorial Limusa. México, D.F., México. 432 pp.

Schutzman, B., A. P. Vovides y B. Dehgan. 1988. Two new species of Zamia (Zamiaceae, Cycadales) from Southern Mexico. Bot. Gaz. 149(3): 347-360.

Webster, G. L. 1994. Synopsis of the genera and suprageneric taxa of Euphorbiaceae. Ann. Missouri Bot. Gard. 81: 33-144.

Wendt, T. 1987a. Las selvas de Uxpanapa, Veracruz-Oaxaca, México: evidencia de refugios florísticos cenozoicos. Anales Inst. Biol. UNAM, Ser. Bot. 58: 29-54.

Wendt, T. 1987b. Plantae Uxpanapae III. A new species of Biophytum (Oxalidaceae) and five genera new for the Mexican flora. Brittonia 39(1): 133-138.

Wendt, T. 1993. Composition, floristic affinities, and origins of the canopy tree flora of the Mexican Atlantic slope rain forests. In: Ramamoorthy, T. P., R. Bye, A. Lot y J. Fa (eds.). Biological diversity of Mexico: origins and distribution. Oxford University Press. Nueva York, USA. pp. 595-680. 
Wurdack, K. J., P. Hoffmann y M. W. Chase. 2005. Molecular phylogenetic analysis of uniovulate Euphorbiacae (Euphorbiaceae sensu stricto) using plastid rbcL and trnL-F DNA sequences. Amer. J. Bot. 92: 1397-1420.

Zamudio, S. 1988. Descubrimiento de Pitcairnia undulata Scheidweiler (Bromeliaceae) en Tabasco, México. Acta Bot. Mex. 2: 5-9. 